\title{
Myocardial Perfusion Imaging in Assessing Risk of Coronary Events in Patients with Systemic Lupus Erythematosus
}

\author{
MANDANA NIKPOUR, DAFNA D. GLADMAN, DOMINIQUE IBAÑEZ, IAN N. BRUCE, ROBERT J. BURNS, \\ and MURRAY B. UROWITZ
}

\begin{abstract}
Objective. Classic risk factors do not fully account for the increased risk of coronary artery disease (CAD) in systemic lupus erythematosus (SLE), making identification of the subset of patients at risk challenging. In this prospective cohort study we investigated whether myocardial perfusion defects in SLE are predictive of CAD events, independently of traditional Framingham risk factors.

Methods. We performed myocardial perfusion imaging in 122 women with SLE who did not have a history of CAD. Patients had clinical and serologic evaluation, and an assessment of cardiac risk factors. They were then followed for the occurrence of CAD events. Cox regression models were used to determine independent predictors of CAD.

Results. Forty-six (37.7\%) patients had perfusion defects. Median followup was 8.7 years, during which $15 \mathrm{CAD}$ events occurred (1 myocardial infarction, 14 angina). Cox modeling showed that myocardial perfusion defects are strongly predictive of CAD [hazard ratio (HR) $13.0,95 \%$ CI 2.8 to $60.1, \mathrm{p}=0.001]$. Although the 10-year Framingham risk score was significantly predictive of CAD (HR 1.8, 95\% CI 1.1 to 2.9, $\mathrm{p}=0.01$ ), the risk scores in groups with normal and abnormal scans were similar to the "low-risk" general population.

Conclusion. In women with SLE, myocardial perfusion defects are strongly and independently predictive of CAD. Our findings suggest that myocardial perfusion imaging to assess risk of future coronary events should be considered in women with SLE. (First Release Jan 15 2009; J Rheumatol 2009;36:288-94; doi:10.3899/jrheum.080776)
\end{abstract}

\author{
Key Indexing Terms: \\ MYOCARDIAL PERFUSION IMAGING \\ CORONARY ARTERY DISEASE \\ WOMEN
}

\section{RISK ASSESSMENT SYSTEMIC LUPUS ERYTHEMATOSUS MYOCARDIAL INFARCTION}

Systemic lupus erythematosus (SLE) is associated with a 5to 10-fold increased risk of angina and myocardial infarction $(\mathrm{MI})^{1-6}$. This increased risk is greatly magnified in

From the University of Toronto Lupus Clinic and the Centre for Prognosis Studies in the Rheumatic Diseases, Toronto Western Hospital, Toronto, Ontario; the Arthritis Research Campaign Epidemiology Unit, University of Manchester, Manchester, UK; and the University of Toronto and the Division of Cardiology, University Health Network, Toronto, Ontario, Canada.

Supported by the Centre for Prognosis Studies in The Rheumatic Diseases, The Smythe Foundation, The Ontario Lupus Association, and The Lupus Society of Alberta. Dr. Nikpour was supported by the Arthritis Centre of Excellence and the Goeff Carr Lupus Fellowhip.

M. Nikpour, MBBS, FRACP, FRCPA; D.D. Gladman, MD, FRCPC; D. Ibañez, MSc; M.B. Urowitz, MD, FRCPC, University of Toronto Lupus Clinic and the Centre for Prognosis Studies in the Rheumatic Diseases, Toronto Western Hospital; I.N. Bruce, MD, FRCP, Arthritis Research Campaign Epidemiology Unit, University of Manchester; R.J. Burns, MD, FRCPC, University of Toronto and the Division of Cardiology, University Health Network.

Dr. Burns is deceased.

Address reprint requests to Dr. M.B. Urowitz, Centre for Prognosis Studies in the Rheumatic Diseases, Toronto Western Hospital,

Room 1E-409, 399 Bathurst Street, Toronto, Ontario M5T 2S8.

E-mail:m.urowitz@utoronto.ca

Accepted for publication September 23, 2008. young women with SLE - those aged 34 to 44 years are over 50 times more likely to have an MI than age-matched peers $^{2}$. Overall, 1 in 10 patients with SLE develops symptomatic or clinical coronary artery disease (CAD), manifesting as angina, MI, or sudden cardiac death ${ }^{1,2,4,7}$. Classic cardiovascular risk factors as defined in the Framingham model do not fully account for the increased risk of CAD in $\mathrm{SLE}^{8,9}$. After controlling for traditional risk factors such as hypertension and hypercholesterolemia, the relative risk of CAD events in patients with SLE is still over 7 times that of controls ${ }^{8}$. Lupus-related factors that confer risk of CAD independently of traditional risk factors remain to be fully elucidated.

Several studies have sought to determine the prevalence and correlates of asymptomatic or subclinical atherosclerosis in SLE ${ }^{10,11}$. Among these, a study by Roman, et al revealed that atherosclerosis as defined by the presence of plaque on carotid ultrasound was more prevalent among patients with SLE than healthy controls (37.1\% vs $15.2 \%$, respectively; $\mathrm{p}<0.001)^{11}$. In multivariate regression analysis, independent predictors of plaque included longer duration of SLE, a higher SLE damage index score, and a lower incidence of the use of immunosuppressives. 
Numerous other studies using various surrogates of underlying atherosclerosis, such as impaired brachial artery flow-mediated vasodilation or coronary artery calcification, have revealed a prevalence of subclinical atherosclerosis in SLE of $30 \%$ to $50 \%$, similar to that reported by Roman and colleagues ${ }^{12-14}$. However, to date there have been no studies that followed patients with SLE long enough to determine the risk factors for subsequent coronary events in patients who have subclinical atherosclerosis.

We have reported abnormal myocardial perfusion imaging in 38\% of women with SLE who did not have a history of clinical $\mathrm{CAD}^{15}$. In the current study our objective was to follow these patients over several years to determine whether myocardial perfusion defects are predictive of subsequent CAD events, independently of traditional Framingham cardiac risk factors.

\section{MATERIALS AND METHODS}

Study population. We recruited consecutive women attending the University of Toronto Lupus Clinic between September 1996 and December 1998. As only 1 in 10 patients with SLE is male and the risk factors for coronary events are potentially different in this subset of patients, only women were recruited to our study ${ }^{16}$. Patients with a history of CAD were excluded. All patients fulfilled 4 or more of the 1971 or 1982 American College of Rheumatology classification criteria for SLE, or had 3 criteria and a typical lesion of SLE on renal or skin biopsy ${ }^{17,18}$.

Written informed consent was obtained from all participants and the University of Toronto Research Ethics Committee approved the study.

Myocardial perfusion imaging. All patients underwent dual-isotope singlephoton emission computed tomographic (SPECT) myocardial perfusion imaging ${ }^{15}$. Briefly, patients received $3.0 \mathrm{mCi}$ of ${ }^{201} \mathrm{Tl}$ (as thallous chloride) as an intravenous bolus at rest. Fifteen minutes after ${ }^{201} \mathrm{Tl}$ administration, patients were positioned supine within a dual-head, fixed $90^{\circ}$-angle SPECT system for rest ${ }^{201} \mathrm{Tl}$ image acquisition. Data were acquired as 60 frames, $25 \mathrm{~s}$ per frame, according to the current conventional clinical protocol. Patients then proceeded immediately to pharmacologic cardiac stress using either dipyridamole $(0.14 \mathrm{mg} / \mathrm{kg} / \mathrm{min}$ intravenously for $4 \mathrm{~min})$ or dobutamine (for patients with asthma or receiving methyl-xanthine compounds, graded infusion of $5,10,20,30$, and then $40 \mu \mathrm{g} / \mathrm{kg} / \mathrm{min}, 3 \mathrm{~min}$ per infusion). An injection of ${ }^{99 \mathrm{~m}} \mathrm{Tc}$-sestamibi (22-25 mCi), and repeat imaging 30 minutes later using the same detector system for a total of 10 minutes, with electrocardiogram (ECG) gating, was performed according to clinical protocol. During and after pharmacologic stress, heart rate, blood pressure, and 12-lead ECG were monitored each minute until these returned to baseline. Constant 3-lead ECG ST segment and rhythm monitoring was performed. Rest ${ }^{201} \mathrm{Tl}$ and ECG-gated stress ${ }^{99 \mathrm{~m}} \mathrm{Tc}$-sestamibi myocardial perfusion images were reconstructed using filtered back projection per routine clinical protocols.

The scans were reported by a single expert, blinded to patients' clinical and laboratory information. The presence of any perfusion defect, regardless of extent, distribution, or reversibility, resulted in a scan being designated "abnormal." Patients were informed of their scan results through their treating physicians, and those with abnormal scans were referred to a cardiologist for further evaluation.

Clinical and laboratory assessment. At the time of myocardial perfusion imaging, patients underwent clinical and laboratory evaluation, including assessment of serologic profile, disease activity using the SLE Disease Activity Index 2000 (SLEDAI-2K), and disease damage using the Systemic Lupus International Collaborating Clinics Damage Index (SLICC-DI) ${ }^{19,20}$. Other prospectively collected data included height and weight, blood pressure, total, high and low density lipoprotein cholesterol (HDL-C and LDL-
C) levels, blood glucose, smoking history, and medications including corticosteroids, antimalarials, immunosuppressives, antihypertensives, and lipid-lowering and hormone replacement therapy (HRT). Exposure to medications was defined categorically as either present or absent, from clinic entry to the time of the scan, or from the time of the scan to CAD event (or last visit). All data were stored and tracked in a dedicated database. During followup, CAD-related events, namely angina and MI, were ascertained and documented ${ }^{21}$. Angina was defined as chest pain of a typical nature, brought on by exertion and relieved with nitrates. MI was defined as typical chest pain with characteristic ECG and enzyme changes. In addition, the occurrence of CAD events was corroborated by a cardiologist.

Statistical analysis. Comparisons between patients with normal and those with abnormal myocardial scans were made using 2-sample $t$ tests or Mann-Whitney tests (in case of non-normal distribution) for continuous variables and by chi-square analysis for categorical variables. Cox proportional hazards regression models were used to determine the independent predictors of CAD events. Results are reported as hazard ratios (HR) with $95 \%$ confidence intervals $(95 \% \mathrm{CI})$. Two-sided $\mathrm{p}$ values $<0.05$ were considered to indicate statistical significance.

The choice of variables for inclusion in the Cox models was based on clinical relevance and significance in univariate analysis. As all participants were female, age and menopausal status were highly correlated and therefore not included in the same model. Thus the variables entered into the model were disease duration at the time of the scan, presence of an abnormal scan, and the Framingham 10-year risk score calculated at the time of the scan. The 10-year Framingham risk score was calculated using the patient's age, systolic blood pressure, smoking status, and total and HDL cholesterol level according to published algorithms ${ }^{22-24}$. On average, lowrisk women below 50 years of age have a 10-year risk score ranging from -2 to 3 inclusive, while low-risk women above 50 years of age have a 10year risk score ranging from 6 to 8 inclusive ${ }^{24}$.

To determine the contribution of individual components of the Framingham risk score to the prediction of CAD events, we ran a second regression model that included the same variables as the original model, except that in lieu of the total Framingham risk score, we included its components as listed above, together with diabetes, LDL-C, and body mass index (BMI), all measured at the time of the scan. Hypertension was defined as systolic blood pressure $>140 \mathrm{~mm} \mathrm{Hg}$ or diastolic blood pressure $>90 \mathrm{~mm} \mathrm{Hg}$ or treatment with antihypertensives ${ }^{25-27}$. Hypercholesterolemia was defined as cholesterol $\geq 5.2 \mathrm{mmol} / \mathrm{l}$ or lipid-lowering therapy ${ }^{28}$. Reduced HDL-C was defined as HDL-C $<0.9 \mathrm{mmol} / \mathrm{I}^{29,30}$. Elevated LDL$\mathrm{C}$ is defined as LDL-C $>3.4 \mathrm{mmol} / \mathrm{1}^{29,30}$. Diabetes was defined as fasting plasma glucose $>7.0 \mathrm{mmol} / 1$ or diabetes therapy ${ }^{30,31}$. BMI is weight in kilograms divided by the square of the height in meters ${ }^{32}$. Statistical analyses were performed using SAS version 9.1 (SAS Institute, Cary, NC, USA).

\section{RESULTS}

In total, 126 SLE patients with no history of CAD underwent myocardial perfusion imaging. There were no notable adverse events related to the procedure. Four patients had followup of less than 6 months after the scan and were excluded. In the remaining 122 patients, the mean (standard deviation; SD) age and disease duration at study were 44.8 (11.0) and 14.3 (9.5) years, respectively. The mean SLEDAI-2K at study was 3.79 (4.45), indicating mild disease activity. The mean SLICC damage score at study was 1.71 (1.84) indicating mild disease-related damage. Fortysix patients (37.7\%) had perfusion defects. Of these, 37 had perfusion defects in one vessel territory, 6 had perfusion defects in 2 vessel territories, and 3 had perfusion defects in 3 vessel territories. Thirty-five patients had reversible 
defects, 5 had fixed defects, and 6 had both fixed and reversible defects. Only one patient had a prior stroke; this patient had a normal cardiac scan and no subsequent coronary event.

Table 1 shows a comparison of demographic and diseaserelated characteristics of patients with normal $(n=76)$ and abnormal $(n=46)$ myocardial scans. Patients with abnormal scans were on average 5.6 years older at the time of the scan than those with normal scans, but had similar race, disease duration, SLEDAI-2K disease activity score, autoantibody profile, and SLICC-DI score. Patients with abnormal scans were more likely to be postmenopausal [24 (52.2\%) vs 24 (31.6\%); $\mathrm{p}=0.02$ ], but did not differ from the group with normal scans in HRT use. The two groups were similar in exposure to corticosteroids, antimalarials, and immunosuppressives from clinic entry to the time of the scan and from the time of the scan to the time of the coronary event (or last visit). The mean (SD) followup time from myocardial perfusion imaging to a CAD event (or last clinic visit) as of May 2007 was 7.5 (2.7) years (median $8.7 \mathrm{yrs).} \mathrm{A} \mathrm{total} \mathrm{of} 15$ $(12.3 \%)$ patients had CAD events, one MI and 14 angina. The mean (SD) time from scan to CAD event was 2.3 (2.3) years (median $1.4 \mathrm{yr}$ ). In Figure 1, Kaplan-Meier survival curves depict accrual of coronary events over time in patients with normal and those with abnormal scans. A substantially greater proportion of patients with abnormal scans had subsequent CAD events [13 (28.3\%) vs $2(2.6 \%)$; p < $0.001]$. Among the 13 patients with abnormal scans that went on to have CAD events, 12 had single-vessel perfusion defects and one had 3 vessel perfusion defects. At the time of myocardial scanning, the mean (SD) 10-year Framingham risk score was significantly higher in those with an abnormal scan [2.39 (0.98) vs 2.01 (1.10); p = 0.006; Table 2]. Hypertension was significantly more com-

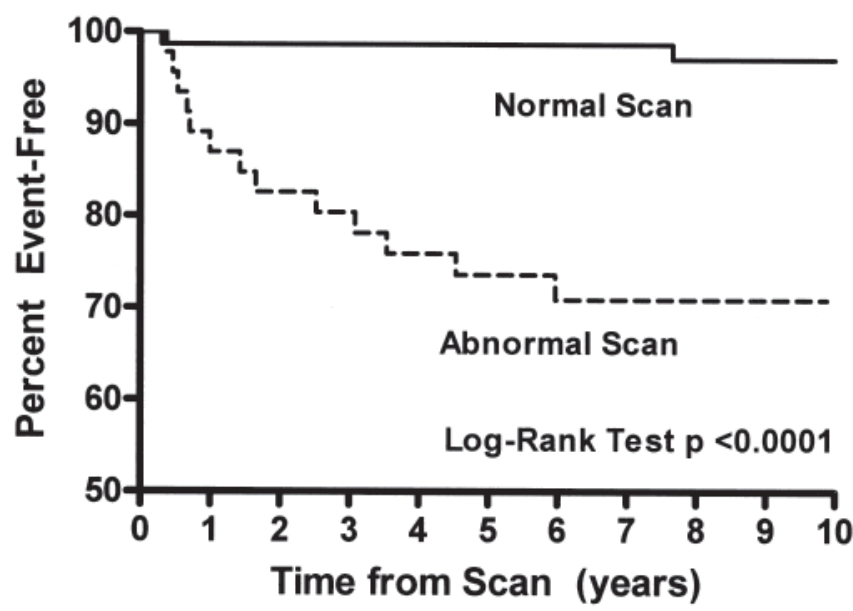

Figure 1. Kaplan-Meier survival curves depicting accrual of coronary events over time in patients with normal and abnormal myocardial perfusion scans. Solid line represents the group with normal myocardial perfusion scans; broken line represents the group with abnormal myocardial perfusion scans. mon among those that had abnormal scans [23 (50.0\%) vs $19(25.0 \%) ; \mathrm{p}=0.005]$, as was use of antihypertensive medications from clinic entry to the time of scan $[27(58.7 \%)$ vs $23(30.3 \%) ; \mathrm{p}=0.002]$. There was no difference in the prevalence of other cardiovascular risk factors or use of lipid-lowering medications between the 2 groups.

The results of the Cox regression analysis of independent predictors of CAD events are presented in Table 3. In the model that included the Framingham risk score but not its individual components, an abnormal myocardial scan was strongly predictive of a future coronary event (HR 13.0, $95 \%$ CI 2.9 to $62.2, \mathrm{p}=0.001)$. In this model, the 10 -year Framingham risk score, calculated at the time of the scan, also had a statistically significant association with $\mathrm{CAD}$ events (HR 1.8, 95\% CI 1.1 to 2.9, p = 0.01).

In the Cox regression model where we included the individual components of the Framingham risk score, but not the score itself, an abnormal scan was still strongly predictive of a subsequent coronary event (HR 9.3, 95\% CI 2.1 to $42.4, p=0.004)$. In this model, hypertension at the time of the scan was also significantly predictive of CAD events (HR 3.8, 95\% CI 1.2 to $12.1, \mathrm{p}=0.03$ ). However, the other components of the Framingham risk score, diabetes, LDL$\mathrm{C}$, and BMI, were not independently related to CAD events.

\section{DISCUSSION}

In this prospective cohort study we found that in women with SLE who did not have a history of CAD, myocardial perfusion defects were strongly and independently predictive of future coronary events. In a time-to-event regression analysis, with a hazard ratio of 13.0 (95\% CI 2.8-60.1, p = 0.001 ), patients with an abnormal cardiac scan were 13 times more likely to have a coronary event than those that had normal scans. We have previously shown that on average, patients tend to have their first coronary event around a decade after diagnosis of $\mathrm{SLE}^{6}$. In this study the mean (SD) disease duration at the time of myocardial imaging was 14.3 (9.5) years. While patients were followed on average 7.5 years subsequent to myocardial perfusion imaging, most coronary events were seen early in followup, with a mean (SD) time to CAD event of 1.9 (2.1) years (median $1.0 \mathrm{yr}$ ). This suggests that myocardial perfusion defects had been present for some time before the scan was performed.

Although we found a statistically significant association between the 10-year Framingham risk score and coronary events, the mean Framingham risk score in both groups, with normal and abnormal scans, was low and similar to scores seen in the "low-risk" general population ${ }^{22,24}$. Therefore this association is of doubtful clinical significance. This finding is consistent with what we have previously shown in the Toronto Risk Factor Study, where the 10year Framingham risk of a CAD-related event was the same in SLE patients and in age-matched population controls ${ }^{30}$.

Among the individual components of the Framingham

Personal non-commercial use only. The Journal of Rheumatology Copyright (c) 2009. All rights reserved. 
Table 1. Demographic and disease-related characteristics of patients with normal and abnormal myocardial perfusion scans.

\begin{tabular}{|c|c|c|c|}
\hline Characteristic & $\begin{array}{c}\text { Normal Scan, } \\
n=76\end{array}$ & $\begin{array}{c}\text { Abnormal Scan, } \\
n=46\end{array}$ & $\mathrm{p}$ \\
\hline Age*, yrs, mean (SD) & $42.7(9.9)$ & $48.3(11.9)$ & 0.007 \\
\hline Disease duration*, yrs, mean (SD) & $13.14(8.7)$ & $16.3(10.5)$ & 0.07 \\
\hline Caucasian, $\mathrm{n}(\%)$ & $55(72.4)$ & $40(87.0)$ & 0.06 \\
\hline Postmenopausal*, n (\%) & $24(31.6)$ & $24(52.2)$ & 0.02 \\
\hline \multicolumn{4}{|l|}{ HRT use, n (\%) } \\
\hline From clinic entry to scan & 17/24 (70.8) & $14 / 24(58.3)$ & 0.37 \\
\hline From scan to CAD event (or last visit) & $25 / 76(32.9)$ & $16 / 46(34.8)$ & 0.83 \\
\hline SLEDAI-2K activity score* ${ }^{* \dagger}$, mean (SD) & $3.7(4.5)$ & $3.9(4.3)$ & 0.87 \\
\hline Elevated anti-DNA antibody* (by radioimmunoassay) & $23(30.3)$ & $16(34.8)$ & 0.60 \\
\hline Low complement* (low C3 or C4) & $21(27.6)$ & $13(28.3)$ & 0.94 \\
\hline Antiphospholipid antibody $* \dagger \dagger$ & $13(17.1)$ & $8(17.4)$ & 0.97 \\
\hline SLICC-DI damage score $* \S$, mean (SD) & $1.57(1.71)$ & $1.93(2.04)$ & 0.30 \\
\hline \multicolumn{4}{|l|}{ Corticosteroid use, n (\%) } \\
\hline From clinic entry to scan & $64(84.2)$ & $38(82.6)$ & 0.82 \\
\hline From scan to CAD event (or last visit) & $57(75.0)$ & $37(80.4)$ & 0.49 \\
\hline \multicolumn{4}{|l|}{ Antimalarial use**, n (\%) } \\
\hline From clinic entry to scan & $55(72.4)$ & $38(82.6)$ & 0.20 \\
\hline From scan to CAD event (or last visit) & $59(77.6)$ & $40(87.0)$ & 0.20 \\
\hline \multicolumn{4}{|l|}{ Immunosuppressive use ${ }^{* * *}, \mathrm{n}(\%)$} \\
\hline From clinic entry to scan & $38(50.0)$ & $25(54.4)$ & 0.64 \\
\hline From scan to CAD event (or last visit) & $39(51.3)$ & $23(50.0)$ & 0.89 \\
\hline
\end{tabular}

* At time of myocardial scan; ${ }^{\dagger}$ scores range from 0 to 105 , higher scores indicating more active disease ${ }^{20}$;

${ }^{\dagger}$ lupus anticoagulant or anticardiolipin antibody; ${ }^{\S}$ scores range from 0 to 46 , higher scores indicating greater disease-related damage ${ }^{19}$; ** chloroquine and hydroxychloroquine; *** methotrexate, azathioprine, mycophenolate mofetil, cyclosporine, and cyclophosphamide. HRT: hormone replacement therapy, CAD: coronary artery disease; SLEDAI-2K: SLE Disease Activity Index 2000; SLICC-DI: Systemic Lupus International Collaborating Clinics-Damage Index.

risk score, only hypertension was independently predictive of CAD events. With a hazard ratio of 3.8 (95\% CI 1.2-12.1, $\mathrm{p}=0.03$ ), patients who had hypertension were almost 4 times more likely to have a coronary event than those that were normotensive. We have previously reported that hypertension is associated with subsequent CAD events in patients with SLE, an association that has been confirmed in the Baltimore Lupus Cohort ${ }^{4,27}$. Collectively, these findings highlight the role of hypertension as a potentially reversible traditional risk factor for coronary events in SLE. The role of nontraditional risk factors, such as high sensitivity Creactive protein and homocysteine, was not assessed in our study.

We used a dual-isotope technique of myocardial perfusion imaging using both ${ }^{201}$ thallium and ${ }^{99 \mathrm{~m}} \mathrm{Tc}$-sestamibi. This method has been shown to have high accuracy for detection and assessment of angiographically significant $\mathrm{CAD}^{33}$. However, of note, other studies using single-isotope ${ }^{99 \mathrm{~m}} \mathrm{Tc}$-sestamibi SPECT myocardial perfusion imaging have revealed a similar prevalence of perfusion defects of around $40 \%$ in asymptomatic patients with $\mathrm{SLE}^{34,35}$. As many patients with SLE have limited exercise capacity due to joint pain or disability, we used pharmacological means of inducing myocardial stress.

Dipyridamole thallium scintigraphy has been shown to be an independent predictor of subsequent coronary events in a large unselected population in whom the majority underwent myocardial perfusion imaging to investigate possible coronary symptoms ${ }^{36}$. Similarly in diabetes mellitus, myocardial perfusion imaging has been shown to have high sensitivity for the detection of angiographically significant coronary stenoses ${ }^{37}$. However, the American Diabetes Association no longer recommends screening diabetic patients with cardiac stress testing or the like, on the grounds that the current status of diabetes as a CAD equivalent already warrants optimized medical therapy ${ }^{38}$. Although it has been suggested that SLE also be considered a CAD equivalent, several key differences distinguish the association between SLE and CAD from that between diabetes and CAD. Most important, traditional risk factors such as hypertension and hypercholesterolemia play a relatively minor role in SLE-related CAD, and to date there have been no studies to show that optimal medical management of these "treatable" risk factors reduces the risk of CAD events in SLE.

In a context where traditional risk factors make a relatively small, albeit potentially reversible contribution to the risk of $\mathrm{CAD}$, and where pathogenic mechanisms of accelerated atherosclerosis in SLE remain largely undefined, myocardial perfusion defects may represent a common final Personal non-commercial use only. The Journal of Rheumatology Copyright (C) 2009. All rights reserved. 
Table 2. Coronary events, cardiac risk factors, and cardiovascular medications in patients with normal and abnormal myocardial scans.

\begin{tabular}{|c|c|c|c|}
\hline Event/Risk Factor & $\begin{array}{c}\text { Normal Scan, } \\
n=76\end{array}$ & $\begin{array}{c}\text { Abnormal Scan, } \\
n=46\end{array}$ & $\mathrm{p}$ \\
\hline New coronary event, $\mathrm{n}(\%)$ & $2(2.6)$ & $13(28.3)$ & $<0.0001$ \\
\hline Ten-year Framingham risk score ${ }^{* \dagger}$, mean (SD) & $2.01(1.10)$ & $2.39(0.98)$ & 0.006 \\
\hline Hypertension*\$, n (\%) & $19(25.0)$ & $23(50.0)$ & 0.005 \\
\hline Systolic blood pressure (mm Hg), mean (SD) & $123.6(18.4)$ & $130.9(18.2)$ & 0.04 \\
\hline Diastolic blood pressure $(\mathrm{mm} \mathrm{Hg})$, mean (SD) & $76.9(9.9)$ & $78.1(7.6)$ & 0.48 \\
\hline Hypercholesterolemia**†, n (\%) & $22(29.0)$ & $17(37.0)$ & 0.36 \\
\hline Total cholesterol $(\mathrm{mmol} / \mathrm{l})^{\mathrm{a}}$, mean $(\mathrm{SD})$ & $4.94(1.16)$ & $5.06(1.09)$ & 0.56 \\
\hline Reduced HDL cholesterol ${ }^{* b}, \mathrm{n}(\%)$ & $2(2.7)$ & $2(4.4)$ & 0.63 \\
\hline HDL cholesterol $(\mathrm{mmol} / \mathrm{l})^{\mathrm{a}}$, mean $(\mathrm{SD})$ & $1.47(0.38)$ & $1.34(0.38)$ & 0.07 \\
\hline Elevated LDL cholesterol*c, n (\%) & $17(23.0)$ & $12(26.7)$ & 0.65 \\
\hline LDL cholesterol $(\mathrm{mmol} / \mathrm{l})^{\mathrm{a}}$, mean $(\mathrm{SD})$ & $2.92(0.98)$ & $2.94(1.30)$ & 0.93 \\
\hline Smoking*, n (\%) & $11(14.7)$ & $6(13.0)$ & 0.80 \\
\hline Diabetes*d, n (\%) & $3(4.0)$ & $2(4.4)$ & 1.00 \\
\hline Body mass index*e & $24.4(5.8)$ & $25.9(6.0)$ & 0.16 \\
\hline \multicolumn{4}{|l|}{ Antihypertensive use $^{\mathrm{f}}, \mathrm{n}(\%)$} \\
\hline From clinic entry to scan & $23(30.3)$ & $27(58.7)$ & 0.002 \\
\hline From scan to CAD event (or last visit) & $42(55.3)$ & $28(60.9)$ & 0.54 \\
\hline \multicolumn{4}{|l|}{ Lipid-lowering medication use ${ }^{\mathrm{g}}, \mathrm{n}(\%)$} \\
\hline From clinic entry to scan & $1 / 3(33.3)$ & $5 / 14(35.7)$ & 1.00 \\
\hline From scan to CAD event (or last visit) & $17(23.3)$ & $9(21.4)$ & 0.82 \\
\hline
\end{tabular}

* At the time of myocardial scan; ${ }^{\dagger}$ calculated using patient's age, systolic blood pressure, smoking status, and total and high density cholesterol level; on average, low-risk women below 50 years of age have a 10 -year risk score ranging from minus 2 to 3 inclusive, while low-risk women above 50 years of age have a 10-year risk score ranging from 6 to 8 inclusive $22-24$; $\$$ defined as systolic blood pressure $\geq 140 \mathrm{~mm} \mathrm{Hg}$ or diastolic blood pressure $\geq 90 \mathrm{~mm} \mathrm{Hg}$ or treatment with antihypertensives ${ }^{25-27}$; $\dagger \geq 5.2 \mathrm{mmol} / \mathrm{l}$ or lipid-lowering therapy ${ }^{28}$ a To convert $\mathrm{mmol} / \mathrm{l}$ to $\mathrm{mg} / \mathrm{l}$ multiply by $38.67 ;{ }^{\mathrm{b}}<0.9 \mathrm{mmol} / \mathrm{I}^{29,30} ;{ }^{\mathrm{c}}>3.4 \mathrm{mmol} / \mathrm{l}^{29,30} ;{ }^{\mathrm{d}}$ defined as fasting plasma glucose $>7.0 \mathrm{mmol} / 1$ or diabetes therapy ${ }^{30,31}$; ${ }^{\mathrm{e}}$ weight in kilogram divided by the square of the height in meters ${ }^{32}$; $\mathrm{f}$ diuretics, beta-blockers, calcium-channel blockers, angiotensin-converting enzyme inhibitors, and angiotensin II receptor blockers; g HMG-CoA reductase inhibitors ("statins").

Table 3. Cox regression analysis of independent predictors of coronary events in patients with systemic lupus erythematosus.

\begin{tabular}{lcc}
\hline Variable & Hazard Ratio (95\% CI) & $\mathrm{p}$ \\
\hline $\begin{array}{l}\text { Disease duration at time of myocardial scan } \\
\text { Abnormal myocardial scan }\end{array}$ & $13.0(2.8-60.1)$ & $\mathrm{NS}$ \\
Ten-year Framingham risk score ${ }^{\dagger}$ at time of myocardial scan & $1.8(1.1,2.9)$ & 0.001 \\
\hline${ }^{\dagger}$ Calculated using patient's age, systolic blood pressure, smoking status, and total and high density cholesterol $^{22-24}$. For definitions of hypertension, hypercholesterolemia, reduced HDL-C, and elevated LDL-C refer to \\
$\begin{array}{l}\text { level } \\
\text { the legend for Table 2. NS: nonsignificant, p }>0.05 .\end{array}$
\end{tabular}

outcome of the interplay between putative and protective factors. We have shown for the first time that myocardial perfusion defects are not only strongly predictive of future coronary events in SLE, but that this association is independent of traditional risk factors. As myocardial perfusion imaging is only minimally invasive and carries a low risk of adverse events, where resources are available, myocardial scanning should be included in the cardiac risk assessment of patients with SLE, including those who have no coronary symptoms. This will enable selection of "high-risk" patients who would potentially benefit from aggressive treatment of classic risk factors where these are present, and from treat- ment with antiplatelet agents and "statins." Although intervention studies are required to determine the role of such drugs in prevention of first-time coronary events in SLE, identification of high-risk patients is the first step toward improving cardiac outcomes in SLE.

We found that age and SLE disease duration were not independently predictive of future coronary events. However, patients with an abnormal scan ranged in age from 21.7 to 71.1 years (median $47.7 \mathrm{yrs}$ ) and had disease durations that ranged from 0.1 to 41.4 years (median $14.8 \mathrm{yrs}$ ). These observations indicate that SLE patients of all ages, even those with relatively short disease duration, should 
undergo myocardial perfusion imaging to assess cardiac risk. Due to the small number of patients in each group, we were unable to perform subgroup analyses to determine whether the extent or reversibility of abnormality on myocardial perfusion imaging has prognostic significance for risk of future coronary events.

A limitation of our study is that due to lack of serial imaging, we were unable to track the progression of perfusion defects over time and are therefore unable to make recommendations as to the frequency with which myocardial perfusion scanning should be repeated in SLE patients that have perfusion defects and those that do not. Further, we have shown that nearly $38 \%$ of patients with SLE who do not have a history of CAD have perfusion defects on myocardial scanning. Yet only $10 \%$ of patients with SLE go on to experience cardiac events ${ }^{1,2,4,7}$. This points to the need for research into novel markers of cardiac risk in SLE that may better define the subset of patients at risk.

This is the first study to link a subclinical measure of coronary artery disease with subsequent clinical events in patients with SLE. Our study has important implications for clinical practice. Our findings suggest that in order to assess risk of future coronary events myocardial perfusion imaging should be considered in women with SLE. Future research should focus on delineating the natural history of perfusion defects and hence the frequency with which scans should be repeated, as well as determining the efficacy of interventions aimed at preventing coronary events in those with perfusion defects.

\section{REFERENCES}

1. Gladman DD, Urowitz MB. Morbidity in systemic lupus erythematosus. J Rheumatol 1987;14 Suppl 13:223-6.

2. Manzi S, Meilahn EN, Rairie JE, et al. Age-specific incidence rates of myocardial infarction and angina in women with systemic lupus erythematosus: comparison with the Framingham Study. Am J Epidemiol 1997;145:408-15.

3. Nikpour M, Urowitz MB, Gladman DD. Premature atherosclerosis in systemic lupus erythematosus. Rheum Dis Clin North Am 2005;31:329-54, vii-viii.

4. Petri M, Perez-Gutthann S, Spence D, Hochberg MC. Risk factors for coronary artery disease in patients with systemic lupus erythematosus. Am J Med 1992;93:513-9.

5. Rubin LA, Urowitz MB, Gladman DD. Mortality in systemic lupus erythematosus: the bimodal pattern revisited. Q J Med 1985;55:87-98.

6. Urowitz MB, Bookman AA, Koehler BE, Gordon DA, Smythe HA, Ogryzlo MA. The bimodal mortality pattern of systemic lupus erythematosus. Am J Med 1976;60:221-5.

7. Abu-Shakra M, Urowitz MB, Gladman DD, Gough J. Mortality studies in systemic lupus erythematosus. Results from a single center. I. Causes of death. J Rheumatol 1995;22:1259-64.

8. Esdaile JM, Abrahamowicz M, Grodzicky T, et al. Traditional Framingham risk factors fail to fully account for accelerated atherosclerosis in systemic lupus erythematosus. Arthritis Rheum 2001;44:2331-7.

9. Rahman P, Urowitz MB, Gladman DD, Bruce IN, Genest J Jr. Contribution of traditional risk factors to coronary artery disease in patients with systemic lupus erythematosus. J Rheumatol 1999;26:2363-8.
10. Manzi S, Selzer F, Sutton-Tyrrell K, et al. Prevalence and risk factors of carotid plaque in women with systemic lupus erythematosus. Arthritis Rheum 1999;42:51-60.

11. Roman MJ, Shanker BA, Davis A, et al. Prevalence and correlates of accelerated atherosclerosis in systemic lupus erythematosus. N Engl J Med 2003;349:2399-406.

12. Asanuma Y, Oeser A, Shintani AK, et al. Premature coronary-artery atherosclerosis in systemic lupus erythematosus. N Engl J Med 2003;349:2407-15.

13. El-Magadmi M, Bodill H, Ahmad Y, et al. Systemic lupus erythematosus: an independent risk factor for endothelial dysfunction in women. Circulation 2004;110:399-404.

14. Lima DS, Sato EI, Lima VC, Miranda F Jr, Hatta FH. Brachial endothelial function is impaired in patients with systemic lupus erythematosus. J Rheumatol 2002;29:292-7.

15. Bruce IN, Burns RJ, Gladman DD, Urowitz MB. Single photon emission computed tomography dual isotope myocardial perfusion imaging in women with systemic lupus erythematosus. I. Prevalence and distribution of abnormalities. J Rheumatol 2000;27:2372-7.

16. Gladman D. Epidemiology of systemic lupus erythematosus. In: Lahita R, editor. Systemic lupus erythematosus. 4th ed. New York: Elsevier Academic Press; 2004:697-709.

17. Lee P, Urowitz MB, Bookman AA, et al. Systemic lupus erythematosus. A review of 110 cases with reference to nephritis, the nervous system, infections, aseptic necrosis and prognosis. Q J Med 1977;46:1-32.

18. Tan EM, Cohen AS, Fries JF, et al. The 1982 revised criteria for the classification of systemic lupus erythematosus. Arthritis Rheum 1982;25:1271-7.

19. Gladman D, Ginzler E, Goldsmith C, et al. The development and initial validation of the Systemic Lupus International Collaborating Clinics/American College of Rheumatology damage index for systemic lupus erythematosus. Arthritis Rheum 1996;39:363-9.

20. Gladman DD, Ibanez D, Urowitz MB. Systemic Lupus Erythematosus Disease Activity Index 2000. J Rheumatol 2002;29:288-91.

21. Alpert JS, Thygesen K, Antman E, Bassand JP. Myocardial infarction redefined - a consensus document of The Joint European Society of Cardiology/American College of Cardiology Committee for the redefinition of myocardial infarction. J Am Coll Cardiol 2000;36:959-69.

22. Fodor JG, Frohlich JJ, Genest JJ Jr, McPherson PR. Recommendations for the management and treatment of dyslipidemia. Report of the Working Group on Hypercholesterolemia and Other Dyslipidemias. CMAJ 2000;162:1441-7.

23. Grundy SM, Pasternak R, Greenland P, Smith S Jr, Fuster V. Assessment of cardiovascular risk by use of multiple-risk-factor assessment equations: a statement for healthcare professionals from the American Heart Association and the American College of Cardiology. Circulation 1999;100:1481-92.

24. Wilson PW, D'Agostino RB, Levy D, Belanger AM, Silbershatz H, Kannel WB. Prediction of coronary heart disease using risk factor categories. Circulation 1998;97:1837-47.

25. Chobanian AV, Bakris GL, Black HR, et al. Seventh report of the Joint National Committee on Prevention, Detection, Evaluation, and Treatment of High Blood Pressure. Hypertension 2003;42:1206-52.

26. Chobanian AV, Bakris GL, Black HR, et al. The Seventh Report of the Joint National Committee on Prevention, Detection, Evaluation, and Treatment of High Blood Pressure: the JNC 7 report. JAMA 2003;289:2560-72.

27. Rahman P, Aguero S, Gladman DD, Hallett D, Urowitz MB. Vascular events in hypertensive patients with systemic lupus erythematosus. Lupus 2000;9:672-5. 
28. Bruce IN, Urowitz MB, Gladman DD, Hallett DC. Natural history of hypercholesterolemia in systemic lupus erythematosus. J Rheumatol 1999;26:2137-43.

29. Third Report of the National Cholesterol Education Program (NCEP) Expert Panel on Detection, Evaluation, and Treatment of High Blood Cholesterol in Adults (Adult Treatment Panel III) fina report. Circulation 2002;106:3143-421.

30. Bruce IN, Urowitz MB, Gladman DD, Ibanez D, Steiner G. Risk factors for coronary heart disease in women with systemic lupus erythematosus: the Toronto Risk Factor Study. Arthritis Rheum 2003;48:3159-67.

31. Diagnosis and classification of diabetes mellitus. Diabetes Care 2006;29 Suppl 1:S43-8.

32. Gray DS, Fujioka K. Use of relative weight and body mass index for the determination of adiposity. J Clin Epidemiol 1991;44:545-50.

33. Matzer L, Kiat H, Wang FP, et al. Pharmacologic stress dual-isotope myocardial perfusion single-photon emission computed tomography. Am Heart J 1994;128:1067-76.

34. Lin CC, Ding HJ, Chen YW, Wang JJ, Ho ST, Kao A. Usefulness of technetium-99m sestamibi myocardial perfusion SPECT in detection of cardiovascular involvement in patients with systemic lupus erythematosus or systemic sclerosis. Int J Cardiol 2003;92:157-61.

35. Sun SS, Shiau YC, Tsai SC, Lin CC, Kao A, Lee CC. The role of technetium-99m sestamibi myocardial perfusion single-photon emission computed tomography (SPECT) in the detection of cardiovascular involvement in systemic lupus erythematosus patients with non-specific chest complaints. Rheumatology Oxford 2001;40:1106-11.

36. Hendel RC, Layden JJ, Leppo JA. Prognostic value of dipyridamole thallium scintigraphy for evaluation of ischemic heart disease. J Am Coll Cardiol 1990;15:109-16.

37. Kang X, Berman DS, Lewin H, et al. Comparative ability of myocardial perfusion single-photon emission computed tomography to detect coronary artery disease in patients with and without diabetes mellitus. Am Heart J 1999;137:949-57.

38. Bax JJ, Young LH, Frye RL, Bonow RO, Steinberg HO, Barrett EJ Screening for coronary artery disease in patients with diabetes. Diabetes Care 2007;30:2729-36. 